\title{
Probabilistic Harmonic Calculation in Distribution Networks with Electric Vehicle Charging Stations
}

\author{
Jianxue Wang, ${ }^{1}$ Yanlin Cui, ${ }^{2}$ and Minghui Zhu ${ }^{1}$ \\ ${ }^{1}$ Department of Electrical Engineering, Xian Jiaotong University, Xi'an, Shaanxi 710049, China \\ ${ }^{2}$ Power Dispatch and Control Center of Guangdong Power Grid Corporation, Guangzhou, Guangdong 510080, China \\ Correspondence should be addressed to Jianxue Wang; jxwang@mail.xjtu.edu.cn
}

Received 25 April 2014; Accepted 28 July 2014; Published 14 August 2014

Academic Editor: Weichao Sun

Copyright (c) 2014 Jianxue Wang et al. This is an open access article distributed under the Creative Commons Attribution License, which permits unrestricted use, distribution, and reproduction in any medium, provided the original work is properly cited.

\begin{abstract}
Integrating EV charging station into power grid will bring impacts on power system, among which the most significant one is the harmonic pollution on distribution networks. Due to the uncertainty of the EV charging process, the harmonic currents brought by EV charging stations have a random nature. This paper proposed a mathematical simulation method for studying the working status of charging stations, which considers influencing factors including random leaving factor, electricity price, and waiting time. Based on the proposed simulation method, the probability distribution of the harmonic currents of EV charging stations is obtained and used in the calculation of the probability harmonic power flow. Then the impacts of EVs and EV charging stations on distribution networks can be analyzed. In the case study, the proposed simulation and analysis method is implemented on the IEEE-34 distribution network. The influences of EV arrival rates, the penetration rate, and the accessing location of EV charging station are also investigated. Results show that this research has good potential in guiding the planning and construction of charging station.
\end{abstract}

\section{Introduction}

Car industry has been developing for decades at a cracking pace. In order to pursue better performance and higher utility of vehicles, many valuable researches have been done to address the issues like adaptive control, mathematical modeling, and fault diagnosis [1-3]. In recent years, the pressure of fossil fuel shortage and other environmental problems leads to high-speed development of electric vehicles (EVs) due to their emission-free characteristic and effective utilization of renewable energy $[4,5]$. The promising trend of EVs attracts plenty of researchers to concentrate on the functioning and the influence of electrical vehicles. Unlike conventional vehicles, the power supply of EVs mainly comes from local power distribution system through EV charging stations [6, 7] and that will lead to impacts on distribution network. In order to study the impacts, the EV charging station should be modeled properly and many factors need to be taken into consideration.

The impacts of EV charging station integration can be divided into two categories basically. The first category contains the influences induced by uncoordinated charging of large amounts of EVs such as voltage deviations, thermal overloads, increase in losses, and decrease in voltage stability $[6,8-10]$. Researches have investigated aspects including the different $\mathrm{EV}$ penetrations on distribution network investment [7], the long-term operating capacity reserve [11], and transformer life [12]. Coordinated charging strategies are proposed [12-15] to promote the distribution gird's ability to hold more EVs without reinforcements, improve voltage profile, and reduce power losses.

The other category is the impacts brought by high rating nonlinear switching devices in EV chargers [16] and the typical one in this category is harmonic pollution. Harmonic pollution will shorten the life span of transformers, reduce the utilization of transmission lines, and interfere with the signals of measuring and protection equipment. Therefore, studying the harmonic pollution brought by EV charging stations is of great importance. Paper [17] establishes a stochastic aggregate harmonic load model to study the harmonic characteristics of EV charger. Paper [18] presents a harmonic simulation method to study the harmonic impact of EVs. Paper [19] 
develops an approach to decrease the harmonics in a high EV penetration grid, and the harmonic load flow is used to model the distorted grid and identify the worst bus based on the typical daily load curve.

In most of the calculation, the worst situation of harmonic of charging station was used. However, the probability of the worst situation is rare in daily life. In most of these studies the harmonic currents are generated by individual EV charger. The random characteristic of charging behavior was not considered in the calculation of charging station harmonic and was hardly consistent with the actual situation in our daily life. Besides, there are limited studies focused on the system level to evaluate the harmonic distortion of charging stations.

In this paper, this problem is investigated from single equipment to the entire system. First, a simplified simulation model of EV charger is used to analyze the harmonic characteristics. Second, based on the queuing theory and charger model, a mathematical simulation method to get the probability distribution of charging station state is proposed, considering the influences of random factors, electricity price, and waiting time. The statistics of the simulation results are performed to obtain the probability distribution of total harmonics. Third, through the probability harmonic power flow, the probability characteristics of probability distribution of charging stations' influences on system harmonics are analyzed. At last, the key factors, including the arrival rate, penetration rate, and the access location of EV charging station, were analyzed based on a modified IEEE-34 system.

\section{The Working State Model of EV Charging Station}

2.1. Model the EV Charger. In order to analyze the harmonic characteristic of EV charging station, the basic working model of a single EV charger needs to be built first. In this paper, we consider a kind of currently the most widely used EV charger. This typical EV charger consists of a three-phase uncontrolled rectifier and a high frequency DC/DC converter which are coupled with a high frequency transformer [4]. The output voltage and current of the charger are controlled by feedback control equipment in the high frequency DC/DC converter. Since elaborately modeling the EV charger is too complicated, it consumes much time for the simulation.

To reduce the complexity of the EV charger model, a simplification proposed in [20] is adopted here, where the high frequency DC/DC converter and battery load are replaced by an equivalent resistance. The simplified equivalent circuit is shown in Figure 1.

Based on Ohm's law, the equivalent resistance can be calculated as

$$
R(t)=\frac{U_{I}}{I_{I}(t)}=\frac{U_{I}^{2}}{P_{I}(t)}=\frac{\eta U_{I}^{2}}{P_{O}(t)},
$$

where $R$ is the equivalent resistance of high frequency $\mathrm{DC} / \mathrm{DC}$ converter, $P_{I}$ is the input power of the high frequency DC/DC converter, $P_{\mathrm{O}}$ is the output power of $\mathrm{EV}$ charger, $\eta$ is the efficiency of high frequency DC/DC converter, $U_{I}$ is

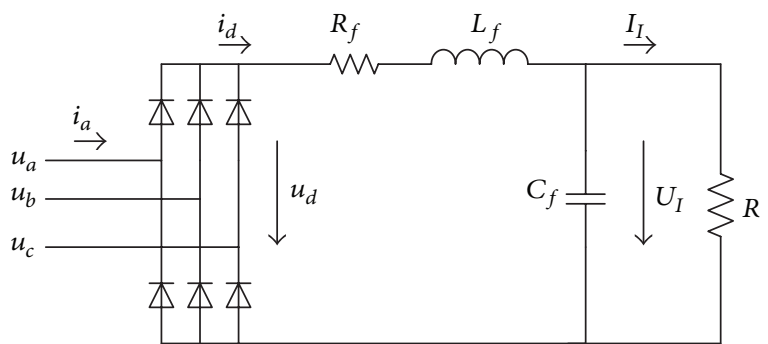

FIGURE 1: The structure diagram of equivalent simplified mode for EV charger [20].

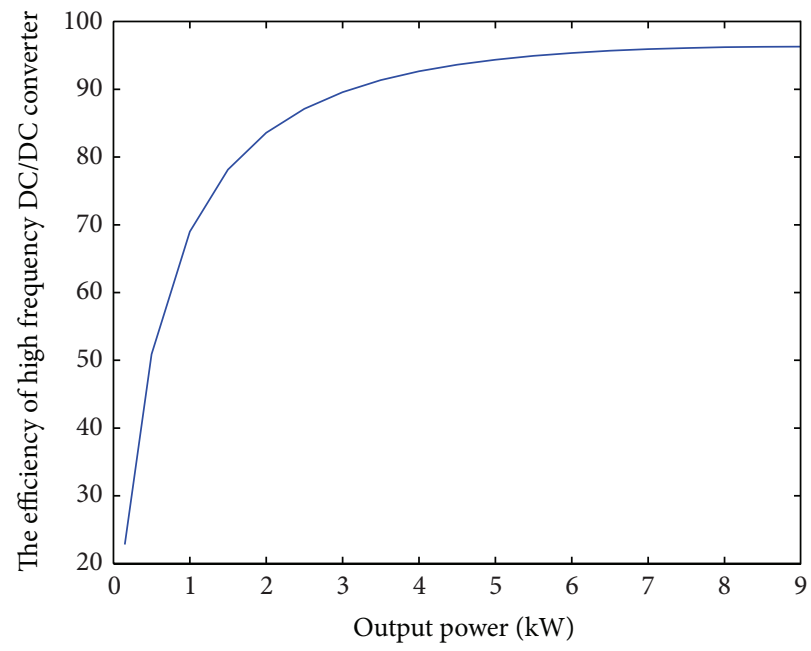

FIGURE 2: The relationship between efficiency and output power.

the output voltage of three-phase uncontrolled rectifier, and $I_{I}$ is the output current of three-phase uncontrolled rectifier.

According to (1), the equivalent resistance should be a function of the charging time to reflect the different output power in different charging stages. The empirical formula of $P_{O}$ during the charging process was obtained through fitting the actual-measured data [20], which can be put as

$$
P_{\mathrm{O}}(t)= \begin{cases}0.79 P_{\mathrm{O} \max } t^{0.048}, & 0<t<t^{\prime}, \\ P_{\text {Omax }} e^{-0.021\left(t-t^{\prime}\right)}, & t^{\prime}<t<t_{\max },\end{cases}
$$

where $P_{O}$ is the output power of EV charger, $t$ is charging time, $t^{\prime}$ is the time spot when charging mode turns from constant current charging to constant voltage charging, $t_{\max }$ is the maximum charging time, and $P_{\mathrm{O} \text { max }}$ is the rated output power.

In [20], $\eta$ was treated as a constant; however, according to the simulation result in MATLAB/Simulink environment, the efficiency of the high frequency DC/DC converter $\eta$ varies with the output power of the EV charger $P_{\mathrm{O}}$. Their relationship is presented in Figure 2.

Using the curve-fitting technique, the expression can be obtained as

$$
\eta\left(P_{\mathrm{O}}\right)=\frac{-0.011+193 P_{\mathrm{O}}}{1+1.785 P_{\mathrm{O}}+0.012 P_{\mathrm{O}}^{2}} .
$$


Through (1)-(3), we can get the value of equivalent resistance at any time spot during charging.

To illustrate the effectiveness of the simplified model in harmonic analysis, a comparison between the original and the simplified model (considering the varying efficiency during charging) has been made through simulating in MAT$\mathrm{LAB} /$ Simulink environment. Simulation results show that the maximum error of the harmonic between the original and the simplified model is merely $0.45 \%$, while the equivalent simplified model costs $40 \%$ less time than the original one. Therefore, the simplified model appears to be more effective and suitable, especially when repetitive calculations are required.

\subsection{Build the Working State Model for an EV Charging} Station. Analysis of the harmonic impact of EV charging stations requires knowing the harmonic currents injection of the charging stations to distribution network. With the EV charger simulation model mentioned in Section 2.1, the harmonic currents that one charger brings to the grid can be obtained in simulation software. The harmonic currents of the entire charging station can be calculated by adding up the harmonic currents generated by every charger in work. Therefore, it is necessary to build the working state model for the EV charging station to acquire the number and the status of working chargers.

To build the working state model, the charging process in the charging station, which depends on EVs' charging behaviors, needs to be studied. Charging behaviors of EVs have much to do with the power conditions of their batteries. Normally, the power condition of a battery is measured by its SOC, which is defined as the available capacity in percentage of full charge capacity [21]. The battery power needs to be adequate to ensure the normal operation of electric vehicles. With the battery power consuming, the SOC will keep dropping and may get down to an overdischarging state [21]. When the battery power drops below a threshold value, the EV cannot work normally and thus needs to get charged at a nearby charging station. The charging process of an EV (as shown in Figure 3) can be modeled as a queuing process based on queuing theory (introduced in [22]), which can be summarized as "first come, first served." The EV that needs charging adds to the tail of the queue when it enters the charging station. If there is no available EV charger, the EV will be waiting for an opening; if there is an available EV charger, it will get charged immediately. The EV will leave when the SOC reaches $100 \%$ or when there are some factors to make it leave early.

In $[23,24]$, the queuing theory is used to describe the charging process and it is proved feasible. In [24], a typical queuing model, $\mathrm{M} / \mathrm{M} / \mathrm{c}$, is used to portray the customer streams of EV charging stations. The typical queuing theory assumes that the inter-arrival and service time are generally subject to exponential distribution. As for EV charging, it is feasible to assume that the interarrival time of two consecutive EVs is exponentially distributed but it will lead to large error to consider that the charging time also obeys exponential distribution. The charging time is a random

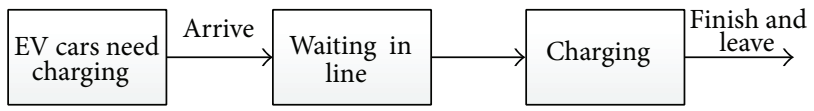

FIGURE 3: The diagram of EVs' charging process.

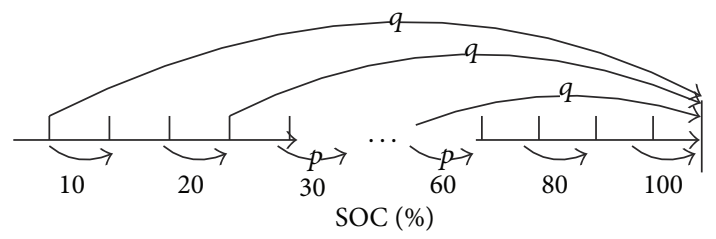

FIGURE 4: The diagram of charging state transition.

variable influenced mainly by the initial SOC, the random leaving factor, the electricity price, and the waiting time, so it does not seem to follow any already known probability distributions. Therefore, the typical queuing model may not describe the charging behavior effectively.

In order to make the model more realistic, this paper adopts a numerical simulating method to study the EV charging process. First, the states of an EV charger should be modeled. The state of charger is determined by the SOC of the battery connected to it, where the range of SOC is from 0 to 100 in percentage. The battery charging process can be treated as a Markov process since the SOC of the next time spot is only related to the current SOC and has nothing to do with the SOC before, which can be put as follows:

$$
\begin{aligned}
P & \left\{\operatorname{SOC}\left(t_{m-1}\right) \subset A \mid \operatorname{SOC}\left(t_{1}\right)=\operatorname{SOC}_{1},\right. \\
& \left.\operatorname{SOC}\left(t_{2}\right)=\operatorname{SOC}_{2}, \ldots, \operatorname{SOC}\left(t_{m}\right)=\operatorname{SOC}_{m}\right\} \\
& =P\left\{\operatorname{SOC}\left(t_{m+1}\right) \subset A \mid \operatorname{SOC}\left(t_{m}\right)=\operatorname{SOC}_{m}\right\},
\end{aligned}
$$

where $t_{m}$ is the current time and $\operatorname{SOC}\left(t_{m}\right)$ is the current state of charge.

In order to make it easier for study, we discretize the domain of SOC's possible values. Figure 4 shows the transition of the states during charging. The SOC of the battery goes towards a higher level over the charging time if the EV does not quit at the middle of charging. If it does quit, the SOC of the battery goes to $100 \%$ directly. It is worth noting that $100 \%$ of SOC here means that the EV leaves and the charger is free. So there are two conditions that a state is treated as a $100 \%$ state. One is that the EV gets fully charged and leaves and the other is that the EV leaves in the middle of charging.

The diagram can be described in

$$
\begin{gathered}
P_{m, m+\Delta \mathrm{SOC}}=p(\mathrm{soc})=1-q(\mathrm{soc}) \\
P_{m, m+\Delta \mathrm{SOC}}=\mathrm{SOC}_{m, m}=0 \\
P_{m, 100}=q(\mathrm{soc}), \\
(0<m<100) .
\end{gathered}
$$




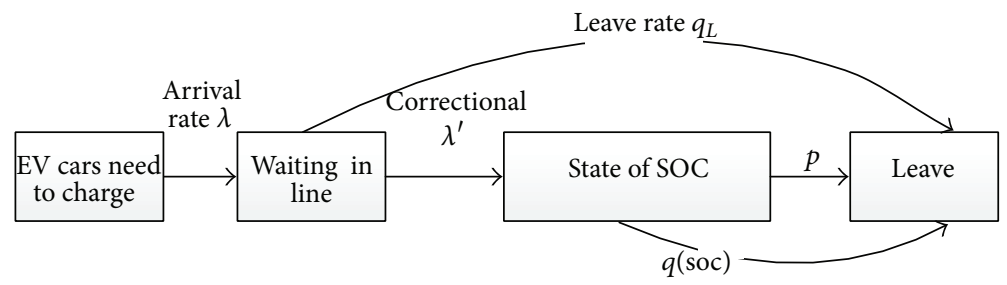

FIGURE 5: The correctional diagram of EVs' charging process.

$P_{i, j}$ means the transition probability from state $i$ to $j$, $\triangle S O C$ is the incremental power in a simulation time unit, and $q$ (soc) is the probability of quitting before the EV is fully charged, which will vary due to the current value of SOC.

To start the simulation, the initial SOC should be given. Setting it to zero or some fixed low value will be inappropriate because the remaining SOC of last time is random. In fact, according to [25], the battery's initial SOC obeys twoparameter $\beta$ distribution, whose probability density function is

$$
\begin{aligned}
f(x ; \alpha, \beta) & =\frac{x^{\alpha-1}(1-x)^{\beta-1}}{\int_{0}^{1} u^{\alpha-1}(1-u)^{\beta-1} d u} \\
& =\frac{\Gamma(\alpha+\beta)}{\Gamma(\alpha) \Gamma(\beta)} x^{\alpha-1}(1-x)^{\beta-1} \\
& =\frac{1}{B(\alpha, \beta)} x^{\alpha-1}(1-x)^{\beta-1}
\end{aligned}
$$

Quitting probability $q$ (soc) needs to be pinned down to complete the model for EV chargers. Generally, it contains 3 components, including random leaving factor, waiting time $q_{\text {time }}$, and electricity price $q_{\text {price }}$.

The typical threshold value SOC' ${ }^{\prime}$ is set to $80 \%$. Leaving earlier before SOC reaches SOC' will do some harm to the battery, so people usually do not leave earlier no matter how long they will wait or how much the electricity costs. When SOC is equal to or greater than the threshold SOC', waiting time factor and electricity cost factor begin to work. The whole process can be illustrated by

$$
q(\mathrm{soc})= \begin{cases}q^{\prime}, & \text { soc }<\mathrm{SOC}^{\prime}, \\ q^{\prime}+q_{\text {time }}+q_{\text {price }}, & \text { soc } \geq \mathrm{SOC}^{\prime} .\end{cases}
$$

Furthermore, $q_{\text {time }}$ is determined not only by the waiting time but also by the difference between the current SOC and the threshold SOC'. With the increasing of SOC, both the waiting time and the difference to SOC' ${ }^{\prime}$ will increase, which leads to a higher waiting time-related quitting probability. In $(8), q_{\text {time }}$ can be thought to be in a linear relationship with the product of the charging time $t$ and the difference between the battery's SOC and SOC' in this study, where time coefficient $k^{\prime}$ is a constant:

$$
q_{\mathrm{time}}= \begin{cases}k^{\prime} t\left(\mathrm{sOc}-\mathrm{SOC}^{\prime}\right), & \text { soc } \geq \mathrm{SOC}^{\prime}, \\ 0, & \text { soc }<\mathrm{SOC}^{\prime}\end{cases}
$$

$q_{\text {price }}$ is also decided by two components. One is the difference between the real-time electricity price $\left(p_{\text {now }}\right)$ and the expected electricity price $\left(P_{E}\right)$, and the other is the difference between SOC and SOC'. The expression is shown in (9), where price coefficient $k^{\prime \prime}$ is a constant:

$$
\begin{aligned}
& q_{\text {price }} \\
& = \begin{cases}k^{\prime \prime}\left(p_{\text {now }}-p_{E}\right)(\mathrm{soc}-\mathrm{SOC}), & \text { soc } \geq \mathrm{SOC}^{\prime}, p_{\text {now }}>P_{E}, \\
0, & \text { soc }<\mathrm{SOC}^{\prime} .\end{cases}
\end{aligned}
$$

Finally, a correction should be made to improve the model of charging process. It is noted that an EV driver may be scared off by a long queue or get impatient waiting in line and then decide to leave before getting the car charged, so the effective arrival rate is a little bit less than the arrival rate. The correctional charging process is demonstrated in Figure 5.

Considering the waiting time, the arrival rate is fixed in (10), where $\lambda$ is the real arrival rate and $\lambda^{\prime}$ is the fixed arrival rate. The directly leaving rate $\left(q_{L}\right)$ is the product of the leaving factor $\left(k_{\text {wait }}\right)$ and the average waiting time $\left(T_{\text {wait }}\right)$ which can be acquired in the simulation of queuing process:

$$
\lambda^{\prime}=\lambda-q_{L}=\lambda-k_{\text {wait }} T_{\text {wait }}
$$

Using the model given above to simulate the activity of each EV charger, the state of the whole charging station can be simulated by the sequential Monte Carlo method. This makes the preparation for the calculation of harmonic distribution.

\subsection{Calculate the Harmonic Distribution of Charging Station.} The harmonic currents of the whole charging station can be calculated by adding up the harmonic currents of all EV chargers. However, the states of chargers are uncertain so the harmonics of the charging station cannot be calculated directly. Using the working state model built in Section 2.2, the states of chargers in an EV charging station can be determined at any time spot; then the simulation model of the entire charging station can be built in simulators to calculate the total harmonics. After simulation, the probability distribution of the charging station can be obtained through the statistical analysis of simulation results.

The simulation model of the charging station is built by the simplified models of EV chargers mentioned in Section 2.1. In each step of the EV charging station's mathematical simulation, all the chargers' output power is determined; thus, the equivalent resistance can be calculated through (1). After that, the value of equivalent resistance can be set in MATLAB/Simulink and then the harmonics of the charging station can be simulated for statistical analysis to acquire 


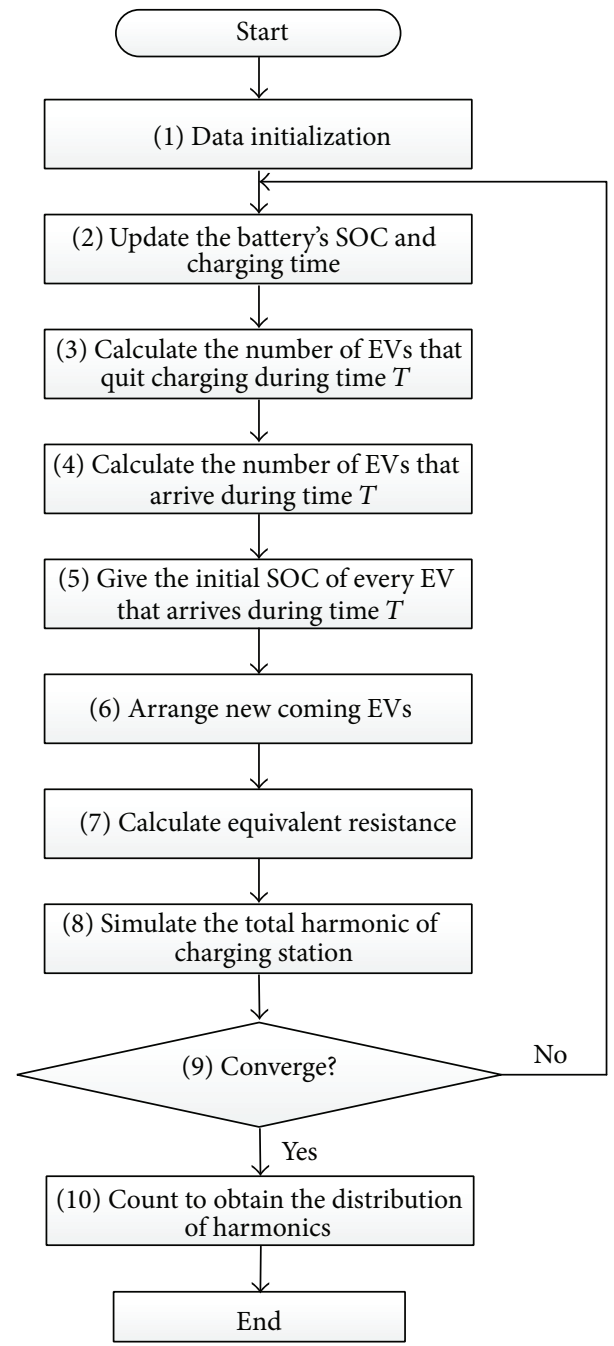

FIGURE 6: Calculating the EV charging station's harmonic distribution.

the probability distribution. Figure 6 illustrates the steps in a flowchart.

Details of the steps in Figure 6 are given as follows.

(1) Initialize the valid data, for example, the simulation step time $(T)$ and the convergence criterion. Then start the mathematical simulation process.

(2) Update the battery's SOC in time $T$ through

$$
\Delta \operatorname{SOC}(t)=\frac{\int_{t}^{t+T} P_{O}(t) d t}{\int_{0}^{t_{\text {all }}} P_{O}(t) d t} \times 100 \%,
$$

where $T$ is the time of simulation step, $t$ is the battery's charging time, and $t_{\text {all }}$ is the total time of a battery's SOC changing from 0 to 100 in percent.

(3) Calculate the number of EVs which quit charging in time $T$, considering the random leaving factor, the price of electricity, and the waiting time.

(4) Calculate the number of EVs which arrive in time $T$ using the correctional arrival rate to increase the calculation accuracy. Then update the number of EV cars in charging station.
(5) Give the initial SOC of each EV which arrives in time $T$ with (1). Then find the corresponding charging time $(t)$ and output power $\left(P_{\mathrm{O}}\right)$.

(6) Arrange the EV cars in the queue for charging according to the "first come, first served" principle.

(7) Calculate the output power by (2) and (3), and then the value of equivalent resistance can be obtained by (1).

(8) Set the resistance parameters of each charger in the simulation model of EV charging station. Then start the simulation and record results of harmonic and total power in an array.

(9) Keep simulation until the probability distribution of charging state meets the convergence criterion that Euclidean distance of probability distributions obtained using different simulation time must be within the range of allowable error, which can be put as

$$
d_{i j}=\sqrt{\sum_{i=1}^{M}\left(x_{i k}-x_{j k}\right)^{2}}<\epsilon
$$

where $x_{i k}, x_{j k}$ stands for the difference of the $k$ th component of random variable $x$ in the $i$ th and $j$ th distributions obtained in different simulation time, $M$ stands for the number of variables, and $\varepsilon$ stands for the allowable error.

(10) Obtain the probability distribution of the charging state and the harmonics.

\section{Probabilistic Harmonic Power Flow of Distribution System Integrating EVs}

The influence of integrating EVs into the distribution network has a probabilistic nature due to the random and uncertain characteristic of EVs. Based on the probability distribution of total harmonic of charging stations, the probability characteristics of charging stations' influences on system harmonics can be analyzed through the probabilistic harmonic power flow $[10,26]$. From the simulation results of harmonics, we find that there is no typical curve that can describe the probability characteristics of charging stations' harmonic. Thus in this research, the Monte Carlo sampling is used to calculate the probabilistic harmonic power flow.

Because the harmonic power brought by EV charging station is much less than the fundamental power in distribution network, the fundamental power flow and the harmonic power flow can be decoupled and calculated, respectively. Newton-Raphson method was used to calculate the fundamental power flow.

The harmonic models of load, transmission line, and transformer adopt the typical harmonic models in [27]. Based on the harmonic model of electrical components and the result of fundamental power flow, $n$ time harmonic admittance matrix $\left(\mathbf{Y}_{\mathbf{n}}\right)$ can be obtained according to the harmonic analysis theory. Harmonic sources are treated as an 
TABLE 1: The detailed information of the charging station.

\begin{tabular}{lccccc}
\hline $\begin{array}{l}\text { Voltage of } \\
\text { network } / \mathrm{kV}\end{array}$ & $\begin{array}{c}\text { The number of } \\
\text { transformers }\end{array}$ & $\begin{array}{c}\text { The ratio of } \\
\text { transformation }\end{array}$ & $\begin{array}{c}\text { The number of } \\
\text { chargers }\end{array}$ & $\begin{array}{c}\text { The total charging } \\
\text { power/kW }\end{array}$ & $\begin{array}{c}\text { The number of } \\
\text { parking spaces }\end{array}$ \\
\hline 10 & 1 & $10 \mathrm{kV} / 400 \mathrm{~V}$ & 35 & 315 \\
\hline
\end{tabular}

TABle 2: Parameters of the EV charger.

\begin{tabular}{lccccc}
\hline Voltage/V & Rated output power/kW & Output voltage/V & Output current/A & $L_{f}(\mathrm{mH})$ & $C_{f}(\mathrm{uF})$ \\
\hline 400 & 9 & 300 & 30 & 1.5 & 2115 \\
\hline
\end{tabular}

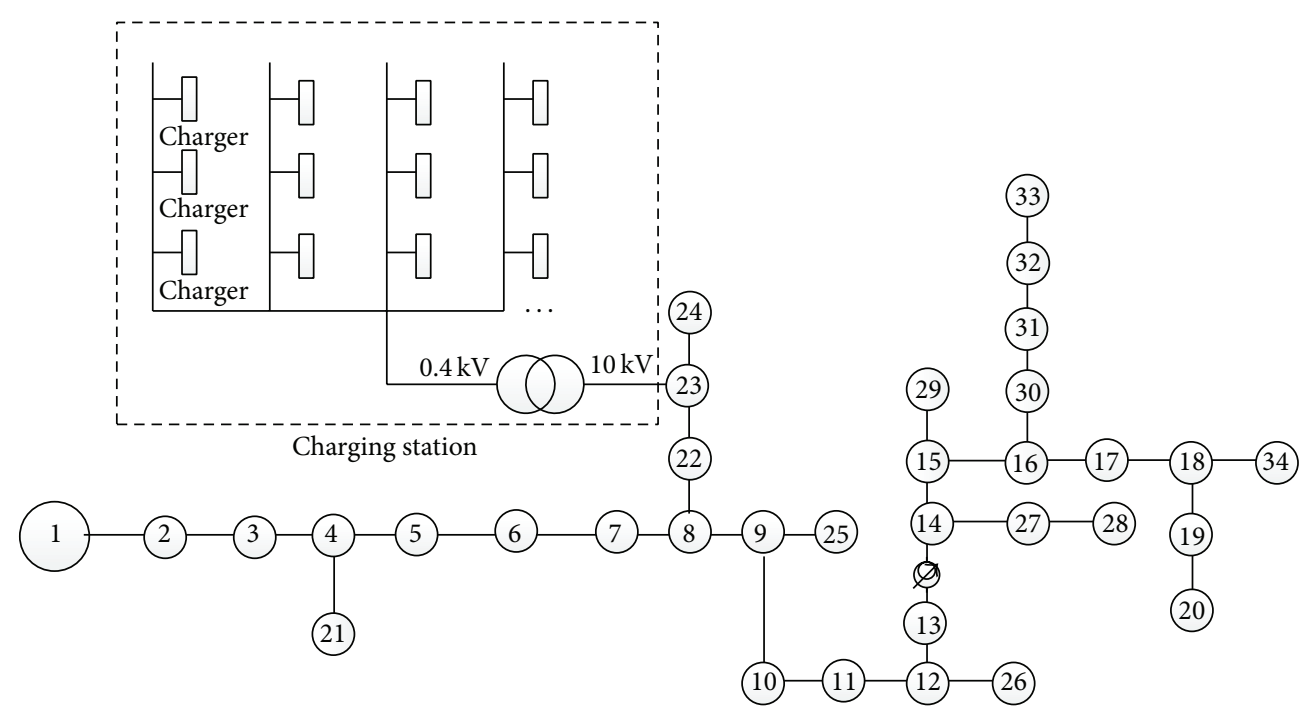

FIgURE 7: The diagram of the modified IEEE-34 test system.

ideal current source, so the $n$ time injection current matrix $\left(\mathbf{I}_{\mathbf{n}}\right)$ can be obtained. The $n$ time harmonic voltage matrix $\left(\mathbf{U}_{\mathbf{n}}\right)$ can be obtained by solving

$$
\mathrm{Y}_{\mathrm{n}} \mathrm{U}_{\mathrm{n}}=\mathrm{I}_{\mathrm{n}} \text {. }
$$

After solving harmonic power flow at each time, the effective voltage $\left(U_{i}\right)$ and total harmonic distortion of voltage $\left(\mathrm{THD}_{v}\right)$ can be calculated through

$$
\begin{gathered}
U_{i}=\sqrt{\sum_{n=1}^{H}\left|U_{1}^{n}\right|^{2}}, \\
\mathrm{THD}_{v}=\left[\frac{\left(\sum_{n=2}^{H}\left|U_{i}^{n}\right|^{2}\right)^{0.5}}{\left|U_{i}^{1}\right|}\right] \times 100 \% .
\end{gathered}
$$

After the calculation of probabilistic harmonic power flow based on Monte Carlo, the probability distribution of effective voltage and $\mathrm{THD}_{v}$ can be acquired. Then the probability characteristics of charging stations' influences on system harmonics under different conditions can be analyzed.

\section{Case Study}

The case study is implemented on a modified IEEE-34 test system [28], which is shown in Figure 7.
TABLE 3: Parameters of the working state model.

\begin{tabular}{lcccccc}
\hline Parameters & $\alpha$ & $\beta$ & $q$ & $k / 10^{-4}$ & $k^{\prime \prime}$ & $k_{\text {wait }} / 10^{-4}$ \\
\hline Value & 2.7 & 5.5 & 0.004 & 1.25 & 0.2 & 1 \\
\hline
\end{tabular}

An EV charging station is connected to the original IEEE-34 test system at Node 23 through a transformer. The penetration power of the EV charging station is set to $15 \%$ in this case. Detailed information of the charging station is listed in Table 1.

The charging time of the EV battery from out-of-power to fully charged is 270 minutes. The parameters of the EV charger are listed in Table 2.

The arrival rate is set to 0.1 during the day and 0.2 at night since fewer people use cars at night and the electricity price is lower. According to the load curve, the load level during the night is lower than other times. The random leaving factor, the waiting time, and electricity price are considered during the daytime. The parameters of the working state model are listed in Table 3.

4.1. The Efficiency Analysis of Working State Model. In order to prove the effectiveness of the model proposed in this paper, the probability distribution of charging station's state is calculated using two different models. The first is the conventional queuing theory model, in which the average service time of 
TABLE 4: Parameters of the probability distribution of station state.

\begin{tabular}{lcccc}
\hline Model & $\begin{array}{c}\text { Expected number of } \\
\text { chargers in work }\end{array}$ & $\begin{array}{c}\text { The number of chargers } \\
\text { with highest possibility }\end{array}$ & $\begin{array}{c}\text { The probability of all } \\
\text { chargers in work }\end{array}$ & $\begin{array}{c}\text { The average length of } \\
\text { the queue }\end{array}$ \\
\hline Typical & 20.3 & 20 & 0.002 & 20.3 \\
Proposed model & 20.3 & 20 & 0.003 & 20.4 \\
\hline
\end{tabular}

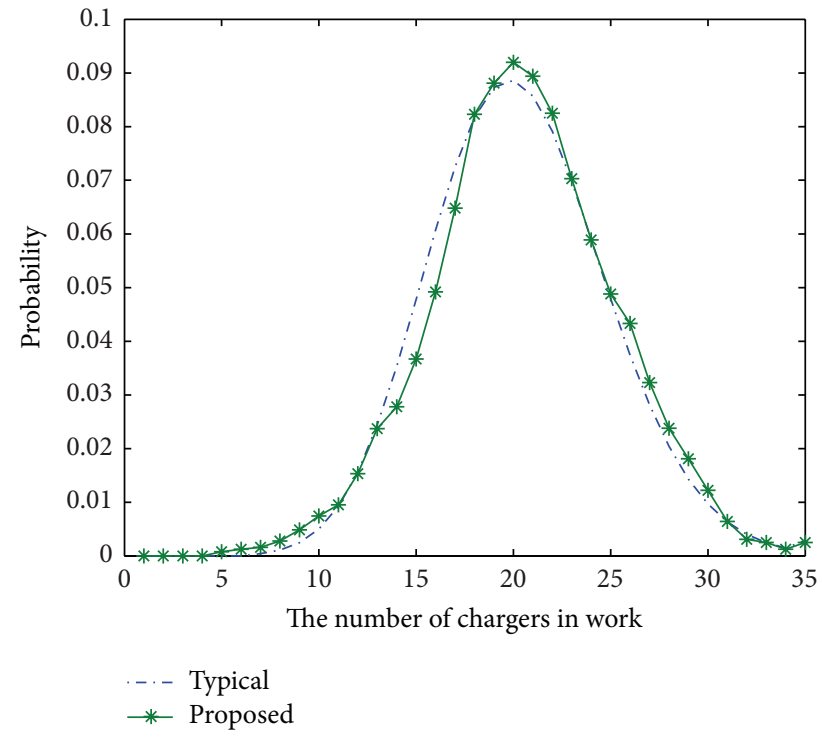

FIGURE 8: The comparison of probability distribution of EV charger in work using typical and proposed model.

charger is 203 minutes. For in the typical model, the random leaving factor, the waiting time, and the price of electricity are not reflected. So in order to prove the effectiveness of the method proposed in this paper, these factors are ignored. The probability distribution of EV charging station's state obtained using two models is shown in Figure 8.

From Figure 8, it is obvious that the probability distribution obtained by the typical model is similar to that by the proposed model.

The parameters of probability distribution of station state are listed in Table 4.

Among the parameters listed in Table 4, the expected number of working chargers and the number of chargers with the highest probability are the same, while the other two parameters are similar in two models. The simulation step time $(T)$ is set to be 3 minutes in this case. If it is set smaller, the value of those similar parameters will get closer. Therefore, the method proposed in this paper can be used to simulate the charging process and calculate the probability distribution of charging station's harmonic.

4.2. Result of Harmonic Probability Power Flow. Based on the working state model of charging station, the distribution of charging station's total power and harmonics can be acquired by doing statistics on simulation results. The probability distributions of charging station's total power during daytime and night are shown in Figures 9 and 10, respectively.

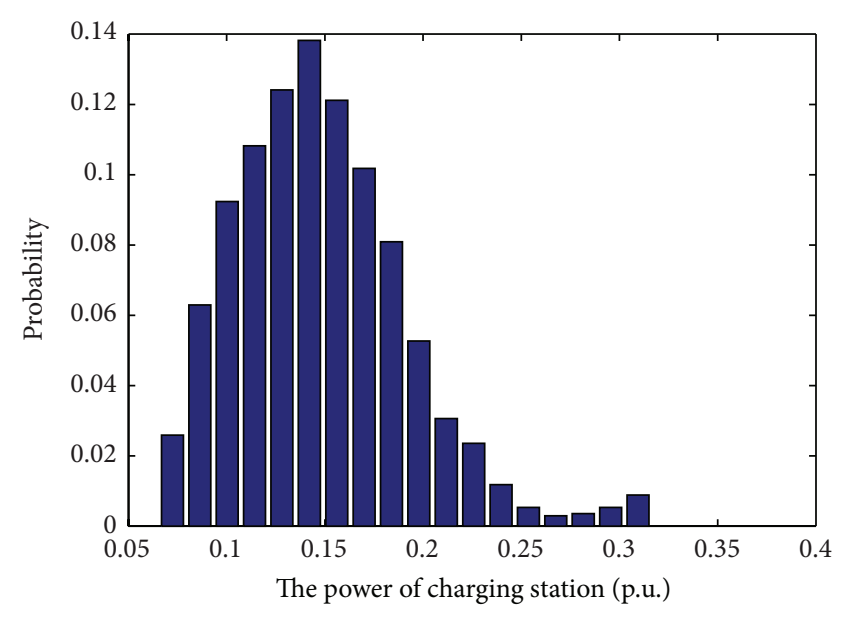

FIGURE 9: The probability distribution of charging station's power during daytime.

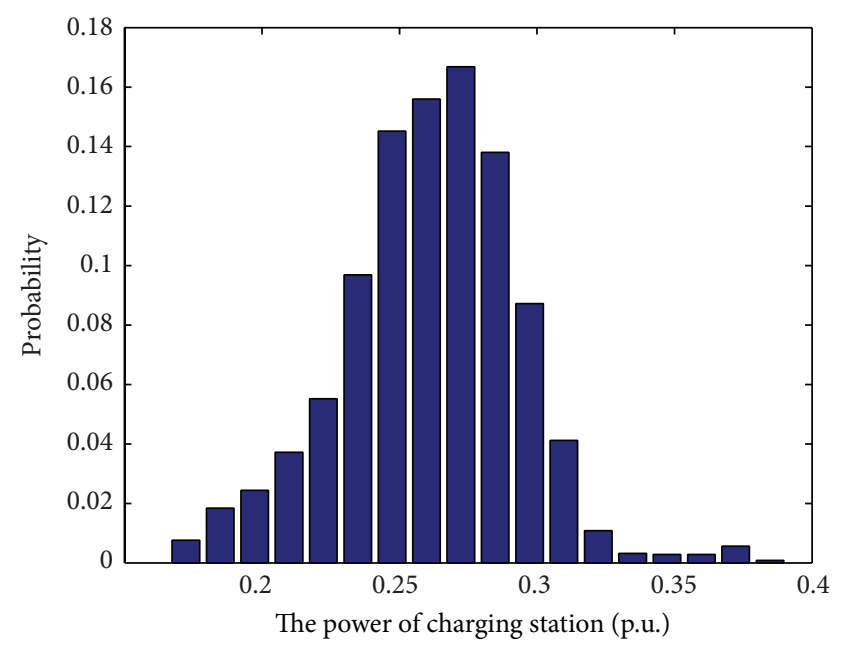

FIGURE 10: The probability distribution of charging station's power during night.

From Figures 9 and 10, it can be noted that the EV charging station is more heavily utilized during night than during daytime.

The distribution of harmonics of charging station can also be acquired. Based on the distribution, the expectation of the current amplitude, the harmonic content, and the expectation of the current angle during day and night are calculated, which are listed in Table 5. It is worth noting that only $6 k \pm 1$ th $(k=1,2, \ldots)$ harmonics are listed because they are the major harmonics of EV charging station. 
TABLE 5: The detailed information of charging station's harmonics.

\begin{tabular}{lccccccc}
\hline \multirow{2}{*}{ Time } & Parameters & & \multicolumn{2}{c}{ Order } & & & \\
& & 5 & 7 & 11 & 13 \\
\hline \multirow{2}{*}{ Daytime } & The expectation of current amplitude $/ 10^{-2}$ p.u. & 3.77 & 1.61 & 0.82 & 0.44 & 0.41 & 0.25 \\
& The harmonic content/\% & 34.2 & 14.6 & 7.5 & 4.0 & 3.7 & 2.3 \\
\hline \multirow{2}{*}{ Night } & The expectation of current amplitude $/ 10^{-2}$ p.u. & 6.45 & 2.13 & 1.47 & 0.74 & 0.66 & 0.45 \\
& The harmonic content/\% & 31.5 & 10.4 & 7.2 & 3.6 & 3.3 & 2.2 \\
\hline
\end{tabular}

TABLE 6: The detailed information of voltage RMS over the range.

\begin{tabular}{|c|c|c|c|c|c|c|c|c|c|c|c|}
\hline Node & 8 & 9 & 10 & 11 & 12 & 13 & 22 & 23 & 24 & 25 & 26 \\
\hline The probability of voltage RMS over the range $/ 10^{-2}$ & 0.01 & 0.27 & 4.88 & 5.50 & 6.73 & 7.70 & 0.03 & 0.03 & 2.07 & 0.27 & 13.69 \\
\hline
\end{tabular}

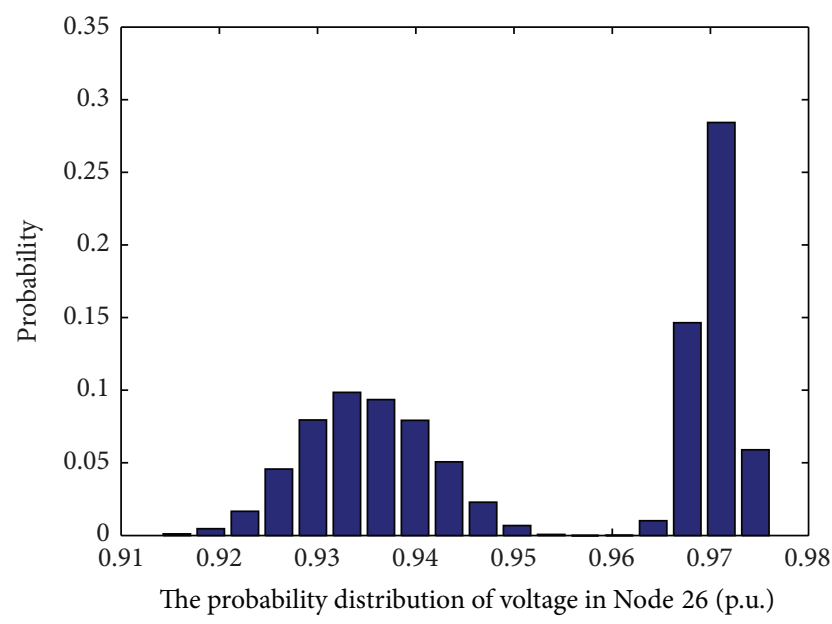

FIGURE 11: The probability distribution of voltage in node 26.

The expectation of current amplitude decreases with the increase of the harmonic order. It is easy to see that the loworder harmonic current accounts for larger proportion than the high-order harmonic. The harmonics with orders higher than 19 are not listed because the amplitudes are too small; thus, the influences are neglectable.

Based on the result of the probabilistic harmonic power flow, the probability distributions of root mean square (RMS) voltages of each node are obtained. The overranging nodes are listed in Table 6.

According to Table 6, there are 11 nodes whose RMS voltages are overrange. Among these nodes, Node 26 has the highest overranging probability. The probability distribution of voltage RMS of Node 26 is shown in Figure 11.

It can be seen that the distribution has a bimodal characteristic, which is due to the peak-valley load during 24 hours.

Based on the result of the probabilistic harmonic power flow, the expected $\mathrm{THD}_{v}$ of each node is calculated, which is shown in Figure 12.

From Figure 12, it can be observed that Node 23, the EV charging station node, and its neighbor node, Node 24, have very high expected $\mathrm{THD}_{v}$. Generally, it is obvious that the expected $\mathrm{THD}_{v}$ of the node near Node 23 is high while the expected $\mathrm{THD}_{v}$ of the node far from Node 23 is low.

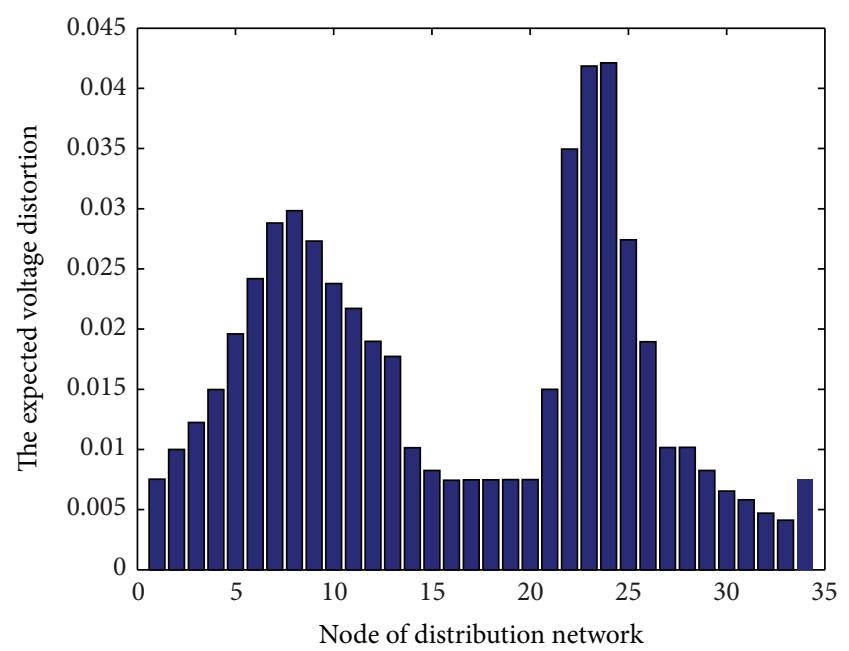

FIGURE 12: The expected voltage distortion of each node.

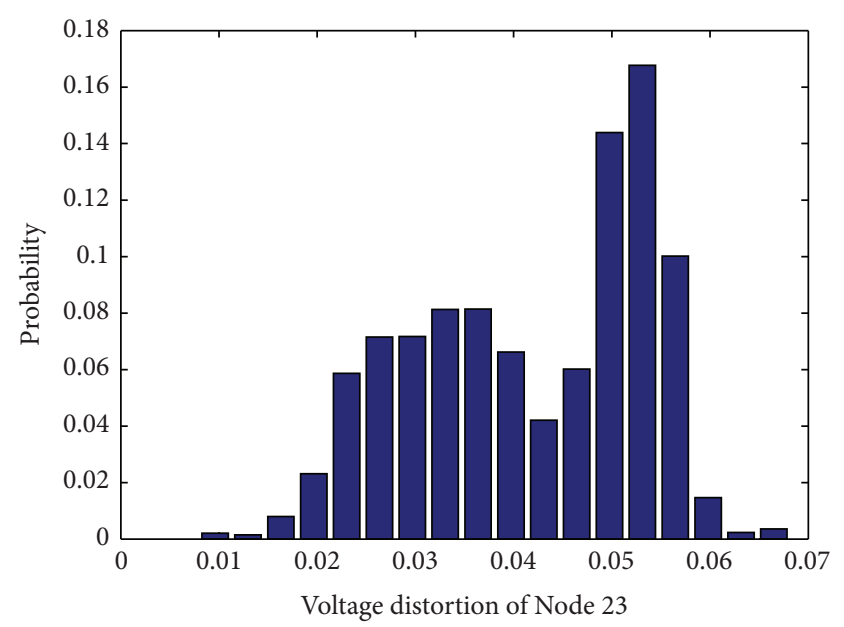

FIGURE 13: The probability distribution of voltage distortion in node 23.

The probability distribution of $\mathrm{THD}_{v}$ of Node 23 is shown in Figure 13.

Table 7 lists the nodes whose $\mathrm{THD}_{v}$ is over range. There are 11 nodes whose probability of $\mathrm{THD}_{v}$ is over the range; 7 of them have an overranging probability that is higher than 
TABLE 7: The detailed information of $\mathrm{THD}_{v}$ over the range.

\begin{tabular}{|c|c|c|c|c|c|c|c|c|c|c|c|}
\hline Node & 5 & 6 & 7 & 8 & 9 & 10 & 11 & 22 & 23 & 24 & 25 \\
\hline The probability of $\mathrm{THD}_{v}$ over the range/\% & 0.4 & 29.2 & 49.2 & 53.6 & 49.2 & 29.6 & 3.8 & 68.1 & 76.1 & 76.1 & 49.2 \\
\hline
\end{tabular}

TABLE 8: The detailed information of $\mathrm{THD}_{v}$ over the range in different arrival rate.

\begin{tabular}{|c|c|c|c|c|c|c|c|c|c|c|c|c|}
\hline Arrival rate & Node & 6 & 7 & 8 & 9 & 10 & 22 & 23 & 24 & 25 & & \\
\hline 0.1 & The probability of $\mathrm{THD}_{v}$ over the range/\% & 0.9 & 5.5 & 9.3 & 3.6 & 0.9 & 25.8 & 53.9 & 53.9 & 3.7 & & \\
\hline Arrival rate & Node & 5 & 6 & 7 & 8 & 9 & 10 & 11 & 22 & 23 & 24 & 25 \\
\hline 0.2 & The probability of $\mathrm{THD}_{v}$ over the range/\% & 0.8 & 43.8 & 93.8 & 96.6 & 89.9 & 45.6 & 3.4 & 100 & 100 & 100 & 89.9 \\
\hline
\end{tabular}

TABLE 9: The detailed information of $\mathrm{THD}_{v}$ over the range when penetration rate is $30 \%$.

\begin{tabular}{lcccccccc}
\hline Node & 4 & 5 & 6 & 7 & 8 & 9 & 10 & 11 \\
\hline The probability of $\mathrm{THD}_{v}$ over the range & 0.048 & 0.454 & 0.700 & 0.889 & 0.889 & 0.889 & 0.747 & 0.668 \\
\hline Node & 12 & 13 & 21 & 22 & 23 & 24 & 25 \\
\hline The probability of $\mathrm{THD}_{v}$ over the range & 0.239 & 0.187 & 0.048 & 0.982 & 0.994 & 0.994 & 0.889 & \\
\hline
\end{tabular}

TABLE 10: The detailed information of $\mathrm{THD}_{v}$ over the range in different accessing location.

\begin{tabular}{|c|c|c|c|c|c|c|c|c|c|c|c|c|c|}
\hline Accessing location & Node & 7 & 8 & 9 & 10 & 11 & 12 & 13 & 22 & 23 & 24 & 25 & 26 \\
\hline 10 & The probability of $\mathrm{THD}_{v}$ over the range/\% & 1.4 & 1.4 & 3.2 & 8 & 5.1 & 1.4 & 0.6 & 1.4 & 1.6 & 1.6 & 3.2 & 1.4 \\
\hline Accessing location & Node & 6 & 7 & 8 & 9 & 10 & 22 & 23 & 24 & 25 & & & \\
\hline 23 & The probability of $\mathrm{THD}_{v}$ over the range/\% & 0.9 & 5.5 & 9.3 & 3.6 & 0.9 & 25.8 & 53.9 & 53.9 & 3.7 & & & \\
\hline Accessing location & Node & 18 & 19 & 20 & 34 & & & & & & & & \\
\hline 34 & The probability of $\mathrm{THD}_{v}$ over the range/\% & 1.4 & 1.4 & 1.4 & 23.7 & & & & & & & & \\
\hline
\end{tabular}

$49 \%$. The result shows that the integration of EV charging station significantly affects the safety and power quality of the distribution network. So filter devices need to be installed to diminish the influence.

4.3. Influence Factors of Charging Station. In this part of research, the influences of the arrival rate, the penetration rate, and the accessing location of EV charging station on the probabilistic harmonic power flow are analyzed as follows.

(1) The Arrival Rate. First, the influence of arrival rate is studied. Calculations have been made when arrival rate is set to different values, 0.1 and 0.2 . The results of the probabilistic harmonic power flow during daytime are listed in Table 8.

With the increase of the arrival rate, the harmonic distortion of the network becomes worse: two new nodes, Node 5 and Node 11, become $\mathrm{THD}_{v}$ overranging nodes. The influenced area becomes larger due to the increase of the arrival rate and so does the $\mathrm{THD}_{v}$ overranging probability. In fact, the overranging probability even reaches $100 \%$ at Nodes 22, 23, and 24. Therefore, it is easy to conclude that the harmonic pollution is more significant when the arrival rate increases.

(2) The Penetration Rate. The harmonic impact on the distribution network is studied during 24 hours when the penetration rate increases to $30 \%$. The results of probabilistic harmonic power flow are listed in Table 9.

Compared to the results in Table 7, the number of $\mathrm{THD}_{v}$ overranging nodes has increased by 4 , the influence area is larger than the original result, and the probability of $\mathrm{THD}_{v}$ over the range changes from 0.761 to 0.994 at Node 23 . It can be seen that the impact on the power quality of the distribution network will be more significant when the penetration rate increases.

(3) Accessing Location. Situations where the EV charging station accesses Nodes 10, 23, and 34 are compared. The results are listed in Table 10.

From the results, two conclusions which are useful in EV charging station planning can be drawn. The influenced area when the EV charging station accesses to Node 10 is larger than that of Node 23. It can be interpreted in a way that Node 10 is the main feeder of distribution network so accessing it will result in a larger influenced area compared with accessing a branch feeder. When accessing location is Node 34, the influenced area and the degree of influence are smaller than that when accessing the other two nodes. That is because Node 34 is close to Node 15 , which is connected with reactive power compensator that has an inhibitory effect on harmonics. Therefore, it can be concluded that it is better to set the charging station close to reactive power compensation devices or away from the main feeder.

\section{Conclusions}

A new method for studying the working status of $\mathrm{EV}$ charging stations is proposed in this paper, while considering the random leaving factor, the electricity price, and the waiting time. This method is proved to be effective through 
the comparison with the conventional queuing theory model. In this paper, this method is used to analyze the characteristics of harmonics from the charging station. The probabilistic harmonic power flow is used to analyze the impact of charging station's harmonics on the distribution network. The proposed model can be directly applied in some other fields that involve charging station's state simulation. In addition, other factors can also be considered in the simulation process. Since the method proposed in this paper is a simulation process, further study will focus on the mathematical theory of the charging behavior.

\section{Conflict of Interests}

The authors declare that there is no conflict of interests regarding the publication of this paper.

\section{Acknowledgment}

This work was supported by National Natural Science Foundation of China (no. 51277141).

\section{References}

[1] W. Sun, H. Gao, and O. Kaynak, "Adaptive backstepping control for active suspension systems with hard constraints," IEEE/ ASME Transactions on Mechatronics, vol. 18, no. 3, pp. 10721079, 2013.

[2] W. Sun, H. Gao, and B. Yao, "Adaptive robust vibration control of full-car active suspensions with electrohydraulic actuators," IEEE Transactions on Control Systems Technology, vol. 21, no. 6, pp. 2417-2422, 2013.

[3] W. Sun, Z. Zhao, and H. Gao, "Saturated adaptive robust control for active suspension systems," IEEE Transactions on Industrial Electronics, vol. 60, no. 9, pp. 3889-3896, 2013.

[4] M. Yilmaz and P. T. Krein, "Review of battery charger topologies, charging power levels, and infrastructure for plug-in electric and hybrid vehicles," IEEE Transactions on Power Electronics, vol. 28, no. 5, pp. 2151-2169, 2013.

[5] C. Shen, P. Shan, and T. Gao, "A comprehensive overview of hybrid electric vehicles," International Journal of Vehicular Technology, vol. 2011, Article ID 571683, 7 pages, 2011.

[6] K. Clement-Nyns, E. Haesen, and J. Driesen, "The impact of charging plug-in hybrid electric vehicles on a residential distribution grid," IEEE Transactions on Power Systems, vol. 25, no. 1, pp. 371-380, 2010.

[7] L. P. Fernández, T. G. S. Román, R. Cossent, C. Domingo, and P. Frías, "Assessment of the impact of plug-in electric vehicles on distribution networks," IEEE Transactions on Power Systems, vol. 26, no. 1, pp. 206-213, 2011.

[8] P. Papadopoulos, S. Skarvelis-Kazakos, I. Grau, L. M. Cipcigan, and N. Jenkins, "Electric vehicles' impact on British distribution networks," IET Electrical Systems in Transportation, vol. 2, no. 3, pp. 91-102, 2012.

[9] C. H. Dharmakeerthi, N. Mithulananthan, and T. K. Saha, "Impact of electric vehicle fast charging on power system voltage stability," International Journal of Electrical Power \& Energy Systems, vol. 57, pp. 241-249, 2014.
[10] S. Deilami, A. S. Masoum, P. S. Moses, and M. A. S. Masoum, "Voltage profile and THD distortion of residential network with high penetration of plug-in electrical vehicles," in Proceedings of the IEEE PES Innovative Smart Grid Technologies Conference Europe (ISGT Europe '10), pp. 1-6, IEEE, October 2010.

[11] L. E. Bremermann, M. Matos, J. A. Peças Lopes et al., "Electric vehicle models for evaluating the security of supply," Electric Power Systems Research, vol. 111, pp. 32-39, 2014.

[12] J. C. Gómez and M. M. Morcos, "Impact of EV battery chargers on the power quality of distribution systems," IEEE Transactions on Power Delivery, vol. 18, no. 3, pp. 975-981, 2003.

[13] S. Deilami, A. S. Masoum, P. S. Moses, and M. A. S. Masoum, "Real-time coordination of plug-in electric vehicle charging in smart grids to minimize power losses and improve voltage profile," IEEE Transactions on Smart Grid, vol. 2, no. 3, pp. 456467, 2011.

[14] P. Richardson, D. Flynn, and A. Keane, "Optimal charging of electric vehicles in low-voltage distribution systems," IEEE Transactions on Power Systems, vol. 27, no. 1, pp. 268-279, 2012.

[15] P. Han, J. Wang, Y. Han, and Y. Li, "Resident plug-in electric vehicle charging modeling and scheduling mechanism in the smart grid," Mathematical Problems in Engineering, vol. 2014, Article ID 540624, 8 pages, 2014.

[16] M. A. S. Masoum, P. S. Moses, and S. Deilami, "Load management in smart grids considering harmonic distortion and transformer derating," in Proceedings of the Innovative Smart Grid Technologies Conference (ISGT '10), pp. 1-7, IEEE, January 2010.

[17] M. T. Au and J. V. Milanović, "Establishing harmonic distortion level of distribution network based on stochastic aggregate harmonic load models," IEEE Transactions on Power Delivery, vol. 22, no. 2, pp. 1086-1092, 2007.

[18] C. Jiang, R. Torquato, D. Salles et al., "Method to assess the power-quality impact of plug-in electric vehicles," IEEE Transactions on Power Delivery, vol. 29, no. 2, pp. 958-965, 2014.

[19] M. A. S. Masoum, S. Deilami, and S. Islam, "Mitigation of harmonics in smart grids with high penetration of plugin electric vehicles," in Proceedings of the IEEE PES General Meeting, pp. 1-6, IEEE, July 2010.

[20] M. Huang, S. F. Huang, and J. C. Jiang, "Harmonic study of electric vehicle chargers," Journal of Beijing Jiaotong University, vol. 32, no. 5, pp. 85-88, 2008.

[21] A. Baba and S. Adachi, "State of charge estimation of HEV/EV battery with series Kalman filter," in Proceedings of the 51st Annual Conference on of the Society of Instrument and Control Engineers of Japan (SICE '12), pp. 845-850, August 2012.

[22] U. N. Bhat, An Introduction to Queueing Theory: Modeling and Analysis in Applications, Springer, New York, NY, USA, 2008.

[23] R. Garcia-Valle and J. G. Vlachogiannis, "Letter to the editor: electric vehicle demand model for load flow studies," Electric Power Components and Systems, vol. 37, no. 5, pp. 577-582, 2009.

[24] G. Li and X. Zhang, "Modeling of plug-in hybrid electric vehicle charging demand in probabilistic power flow calculations," IEEE Transactions on Smart Grid, vol. 3, no. 1, pp. 492-499, 2012.

[25] W. Su and M. Chow, "Performance evaluation of an EDA-based large-scale plug-in hybrid electric vehicle charging algorithm," IEEE Transactions on Smart Grid, vol. 3, no. 1, pp. 308-315, 2012.

[26] A. A. Romero, H. C. Zini, G. Rattá, and R. Dib, "Harmonic loadflow approach based on the possibility theory," IET Generation, Transmission and Distribution, vol. 5, no. 4, pp. 393-404, 2011. 
[27] P. F. Ribeiro, Time-Varying Waveform Distortions in Power Systems, Wiley, 2009.

[28] W. H. Kersting, "Radial distribution test feeders," in Proceedings of the IEEE Power Engineering Society Winter Meeting, pp. 908912, February 2001. 


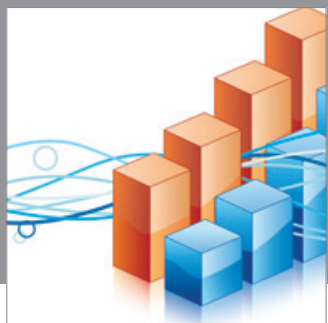

Advances in

Operations Research

mansans

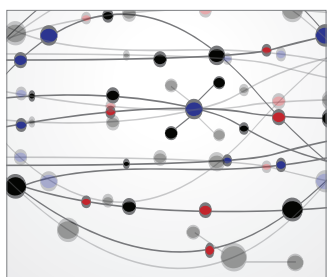

The Scientific World Journal
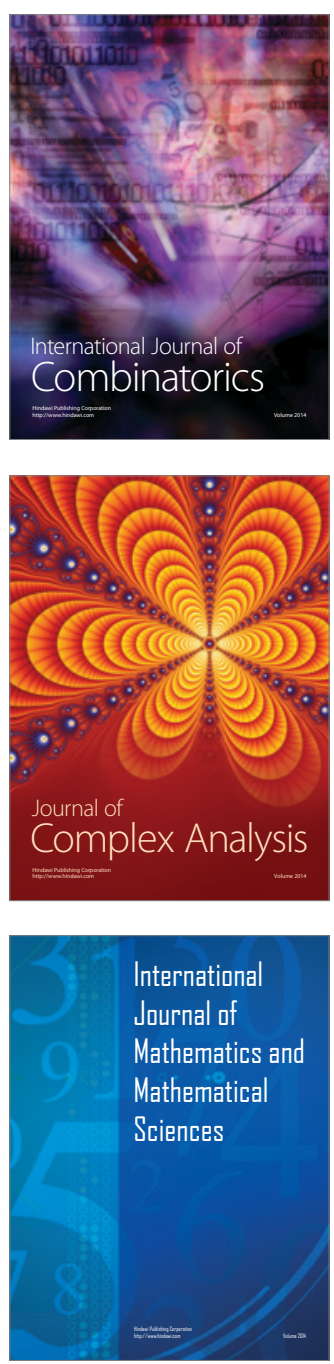
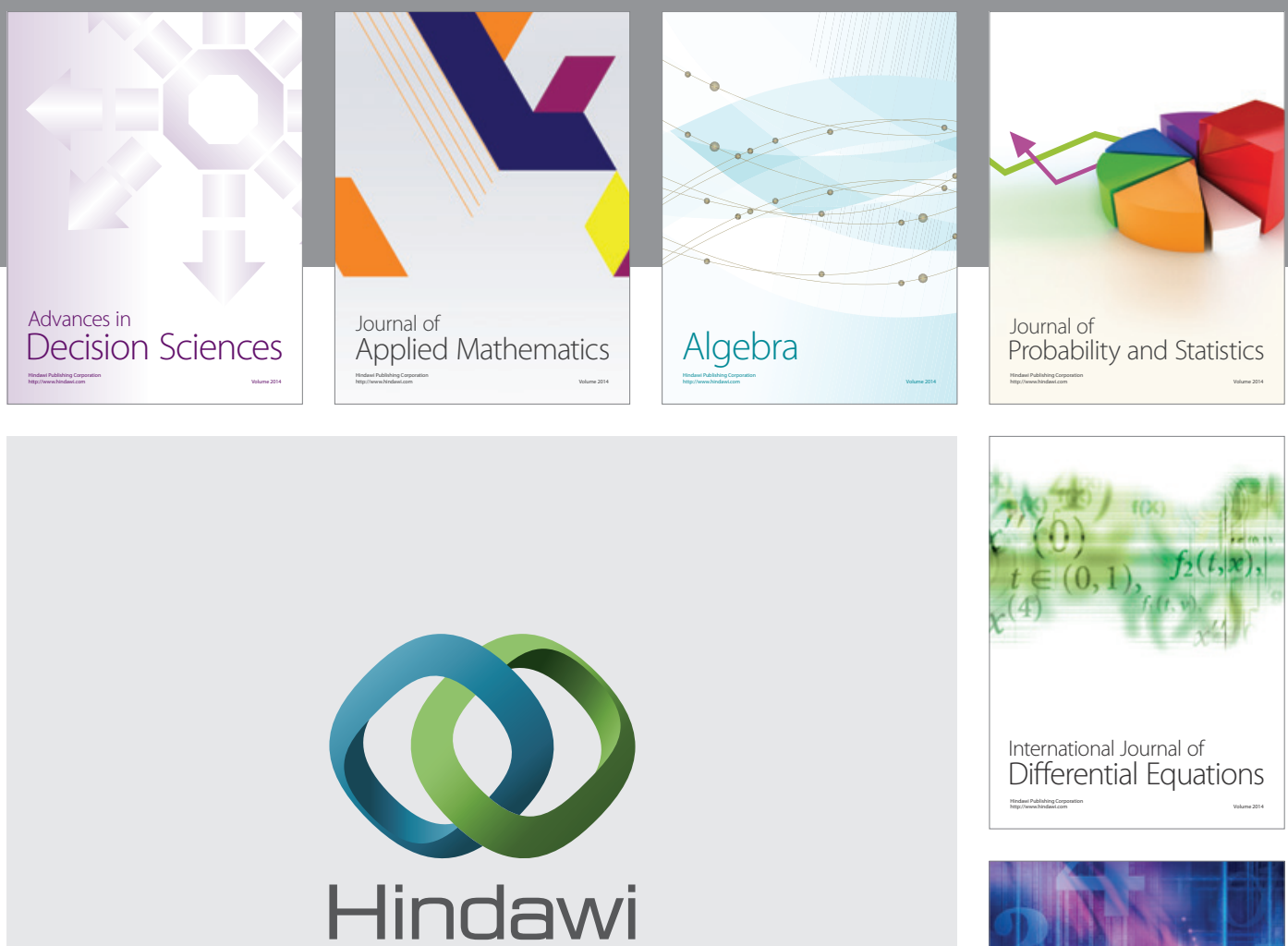

Submit your manuscripts at http://www.hindawi.com
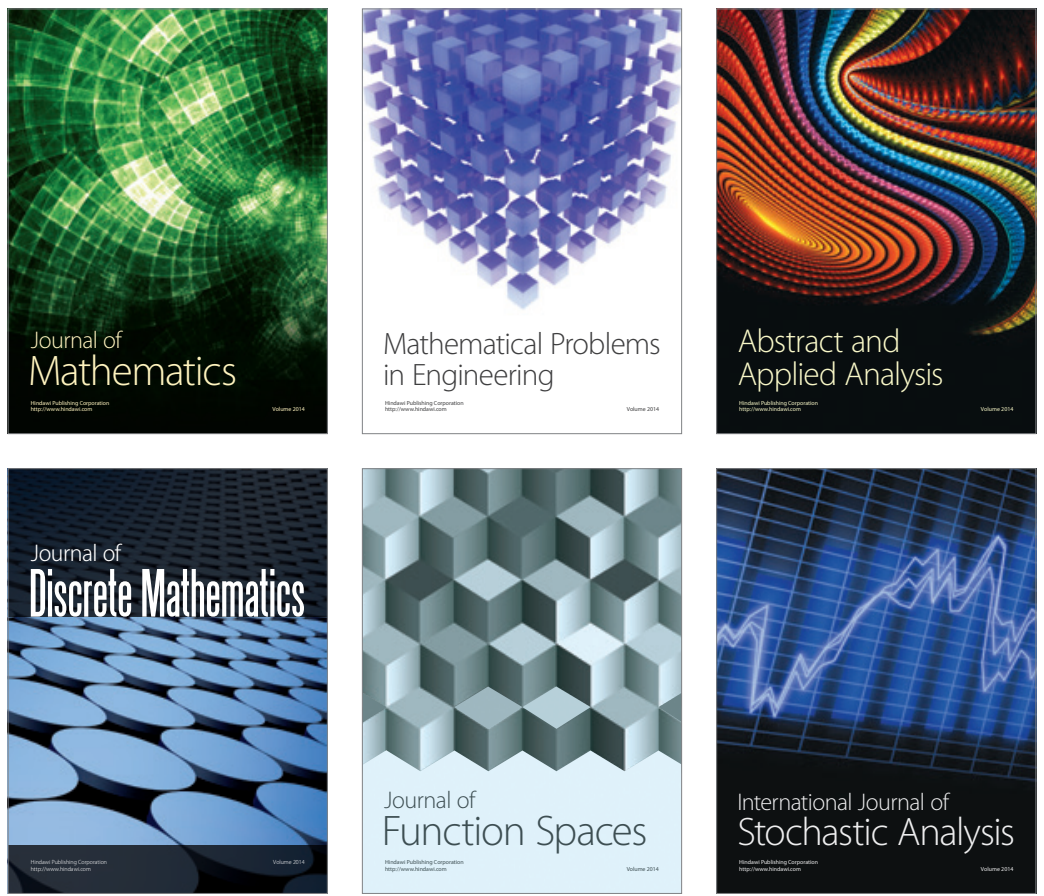

Journal of

Function Spaces

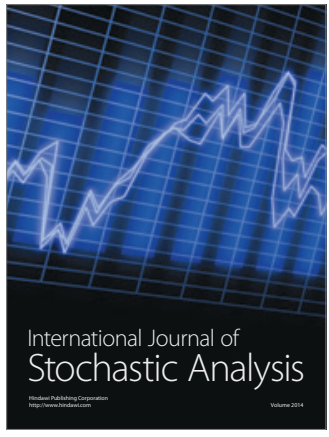

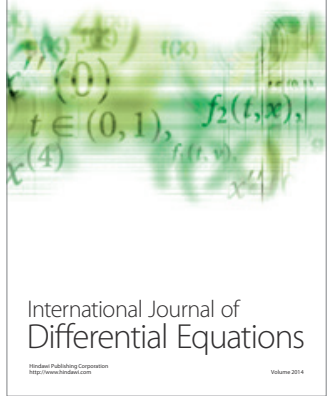
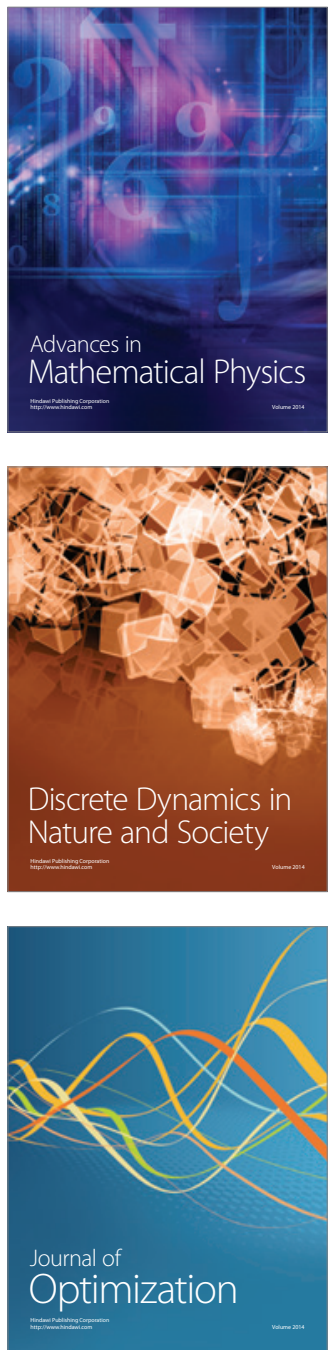